\title{
Stability and Complexity of Spatially Extended Two-species Competition
}

\author{
Ricard V. Soléł, Jordi Bascompteł AND Joaquim VAlls $\dagger$ \\ Complex Systems Research Group, †Departament de Fisica i Enginyeria \\ Nuclear, Universitat Politècnica de Catalunya, Pau Gargallo 5 and \\ $\ddagger$ Departament d’Ecologia, Universitat de Barcelona, Diagonal 645,08028 \\ Barcelona, Spain
}

(Received on 1 February 1992, Accepted on 27 March 1992)

Lotka \& Volterra's studies showed the dynamics of two species in competition. Although it is very simple, the model has not been improved until recently. As Margalef (1980) pointed out, space must be taken into account in all fundamental aspects of ecological organization. On the other hand, in the last few years unexpected results on non-linear dynamical systems have changed our view of complexity. In this paper we explore the spatiotemporal behaviour of a two-species competition coupled map lattice. The coexistence of the two competitors is demonstrated although they have high interspecific competition coefficients. This coexistence is closely related with spatial segregation and the formation of a well-defined Turing-like structure. Moreover, the patches observed can have a large influence on the temporal dynamics. Some implications for population extinction and for the competitive exclusion principle are also discussed.

\section{Introduction}

Lotka (1925) and Volterra (1926) started the study of competition models with the following system of differential equations:

$$
\begin{aligned}
& \frac{\mathrm{d} X}{\mathrm{~d} t}=r_{1} X \frac{\left(K_{1}-X-\alpha Y\right)}{K_{1}} \\
& \frac{\mathrm{d} Y}{\mathrm{~d} t}=r_{2} Y \frac{\left(K_{2}-Y-\beta X\right)}{K_{2}}
\end{aligned}
$$

$X, Y$ being the population sizes of the two species. $r_{1}$ and $r_{2}$ are growth rates, $K_{1}$ and $K_{2}$ are the carrying capacities and $\alpha$ and $\beta$ are the interspecific competition coefficients, a kind of equivalence from one species to the other.

The analytic solution to model (1) shows the existence of three non-trivial fixed points: $P=\left\{\left(X^{*}, Y^{*}\right),\left(K_{1}, 0\right),\left(0, K_{2}\right)\right\}$. The coexistence of the two populations is only possible if $\alpha \beta<1$, i.e. if the interspecific competition pressures are lower than the intraspecific. In other words, when $\alpha \beta>1$ the system evolves toward the extinction of one of the species, depending on initial conditions. This competitive exclusion was soon confirmed by laboratory experiments in which conditions were spatially homogeneous. Otherwise, natural observations and substitutive experiments in the field 
created the core of the ecological niche theory (for a review see, for example, Begon \& Mortimer, 1986).

On the other hand, when space is considered, new, unexpected results appear. Thus, the reaction-diffusion mathematical models can give spatial structures through Turing symmetry-breaking instabilities (Nicolis \& Prigogine, 1977; Murray, 1989). As pointed out by Turing (1952), they can model the problem of pattern formation in developmental biology. These Turing structures that emerge from the interaction between an "activator" and an "inhibitor" that diffuse at differential rates, are also used in the description of the structures in space in ecology (Segel \& Jackson, 1972). An equivalent approach is that based on the Coupled Map Lattice (CML) formalism, that describes the spatiotemporal organization of a continuous variable of state through a discrete time and space. The CML has been applied to the study of turbulence and the structural stability properties of spatiotemporal chaos (Kaneko, 1990), and recently to the modelling of ecosystems (Sole \& Valls, 1991). In a parallel way, the global persistence of populations is shown despite local highly unstable dynamics when spatial degrees of freedom are introduced (Taylor, 1990; Sabelis et al., 1991; Solé \& Valls, 1992). However, part of modern theoretical ecology is still working with Lotka-Volterra-like models. This kind of ecological model, as pointed out by Margalef (1986), accepts that "everything happens at a point, without space, a curious view to hold after centuries of making fun about how many angels can dance on the tip of a pin".

\section{Two-dimensional Competition Discrete Map}

Let us begin by showing the following two-dimensional map which is applied to the study of species competition with non-overlapped generations:

$$
\begin{aligned}
& x_{n+1}=\mu_{1} x_{n}\left(1-x_{n}-\beta_{1} y_{n}\right) \\
& y_{n+1}=\mu_{2} y_{n}\left(1-y_{n}-\beta_{2} x_{n}\right) .
\end{aligned}
$$

Here $x_{n}, y_{n}$ are the population sizes at a given discrete time $n, \mu_{1,2}$ are growth rates and $\beta_{1,2}$ competition rates. Analytically, this system has three non-trivial steady states: exclusion points, $P_{1}=\left(1-1 / \mu_{1}, 0\right), P_{2}=\left(0,1-1 / \mu_{2}\right)$ and a coexistence point $P_{3}=\left(x^{*}, y^{*}\right)$ being: $x^{*}=\left(1-1 / \mu_{1}\right) /\left(1+\beta_{1}\right)$ and $y^{*}=\left(1-1 / \mu_{2}\right) /\left(1+\beta_{2}\right)$.

Here we will consider the case of ecologically identical species, i.e. $\mu_{1,2}=\mu$ and $\beta_{1,2}=\beta$. We have selected the most symmetrical case, but if coexistence is possible here, it will be easily found in some ecological interaction involving real species, where the asymmetries are inevitable. This symmetry in the equation's structure will be discussed in the next section.

It can be proved that the coexistence point is unstable for $\beta>1$ for small perturbations, leading to competitive exclusion. This is in agreement with the analytical properties of Lotka-Volterra's model and with the competitive exclusion theory.

The community matrix is here defined as:

$$
\Gamma=\left(\begin{array}{ll}
\partial_{x} F^{(1)} & \partial_{y} F^{(1)} \\
\partial_{x} F^{(2)} & \partial_{y} F^{(2)}
\end{array}\right)
$$


i.e. we have

$$
\Gamma=\left(\begin{array}{cc}
\mu(1-2 x-\beta y) & -\mu \beta x \\
-\mu \beta y & \mu(1-2 y-\beta x)
\end{array}\right)
$$

For $P_{1}=\left(1-1 / \mu_{1}, 0\right)\left(\right.$ or $\left.P_{2}=\left(0,1-1 / \mu_{2}\right)\right), \Gamma$ will be:

$$
\Gamma\left(P_{1}\right)=\left(\begin{array}{cc}
2-\mu & \beta(1-\mu) \\
0 & \mu(1-\beta)+\beta
\end{array}\right)
$$

which has an associated eigenvalue equation $\left|\Gamma\left(P_{1}\right)-\lambda \mathbf{I}\right|=0$ with two solutions given by $\lambda_{+}=2-\mu$ and $\lambda_{-}=\beta+\mu(1-\beta)$. The attractor will be stable provided that $\left|\lambda_{ \pm}\right|<1$, for both eigenvalues. This condition leads to a stability domain given by

$$
S\left(P_{1}\right)=\{(\mu, \beta) \mid \beta>1 ; \mu \in(1,3)\}
$$

An equivalent result is obtained for the second exclusion point.

For the coexistence attractor, i.e. $P_{3}=\left(x^{*}, y^{*}\right)$, we have an associated matrix

$$
\Gamma\left(P_{3}\right)=\left(\begin{array}{ll}
\Lambda & \alpha \\
\alpha & \Lambda
\end{array}\right)
$$

for which $\Lambda=\mu\left[1-(2+\beta) x^{*}\right]$ and $\alpha=-\mu \beta x^{*}$. The corresponding eigenvalues are now $\lambda_{+}=2-\mu$ and $\lambda=\mu[1-2(1-1 / \mu) /(1+\beta)]$. Now the linear stability analysis gives a new stability domain given by:

$$
S\left(P_{3}\right)=\{(\mu, \beta) \mid 0<\beta<1 ; \mu \in(1,3)\} .
$$

We can see that $\beta$ is a key parameter here in controlling the stability of solutions. For $\beta<1$, the coexistence point is stable and, simultaneously, exclusion points become unstable. In both cases, another kind of instability appears for $\mu_{c}>3$, in which a pitchfork bifurcation takes place (as it is well known for the logistic map). In such a situation, we still have coexistence or exclusion but with a periodic oscillation around the critical point. Chaotic dynamics takes place for $\mu>\mu_{\infty}=$ 3.569 ... after a period doubling bifurcation scenario.

While the Lotka-Volterra competition model [(1a) and (1b)] can only approach the equilibrium point monotonically and can never exhibit oscillatory behaviour, discrete counterparts permit oscillatory damping as well as limit cycles and chaos (Hassell \& Comins, 1976). Our model confirms this richness of dynamical behaviour. In this context, chaos theory has changed our understanding of complexity. Chaotic systems are entirely deterministic (without random inputs) in spite of showing nonperiodic (noise-like) motion. Furthermore, they exhibit sensitive dependence on initial conditions, i.e. the fact that nearby trajectories separate exponentially. The 
more chaotic a system is, the greater the rate of divergence. The measure of this degree of "stretching" is given by the computation of the largest Lyapunov exponent $\left(\lambda_{m}\right)$ :

$$
\lambda_{n}=\frac{1}{r \tau}\left\{\sum_{t=1}^{r} \operatorname{Ln}(\Theta(t, \tau))\right\}
$$

$r$ being the number of points sampled and

$$
\Theta(t, \tau)=\frac{\left\|X(t+\tau)-X^{\prime}(t+\tau)\right\|}{\left\|X(t)-X^{\prime}(t)\right\|} .
$$

There is one Lyapunov exponent for each dimension of the phase space. Thus, a dynamical system is called chaotic if at least one Lyapunov exponent is positive. A negative exponent means that there is convergence of different trajectories in that direction of phase space, while a zero value indicates periodicity (there is neither convergence nor divergence). The largest Lyapunov exponent for different parameter values is shown in Fig. 1, indicating the existence of different stationary, periodic and chaotic attractors for model (2).

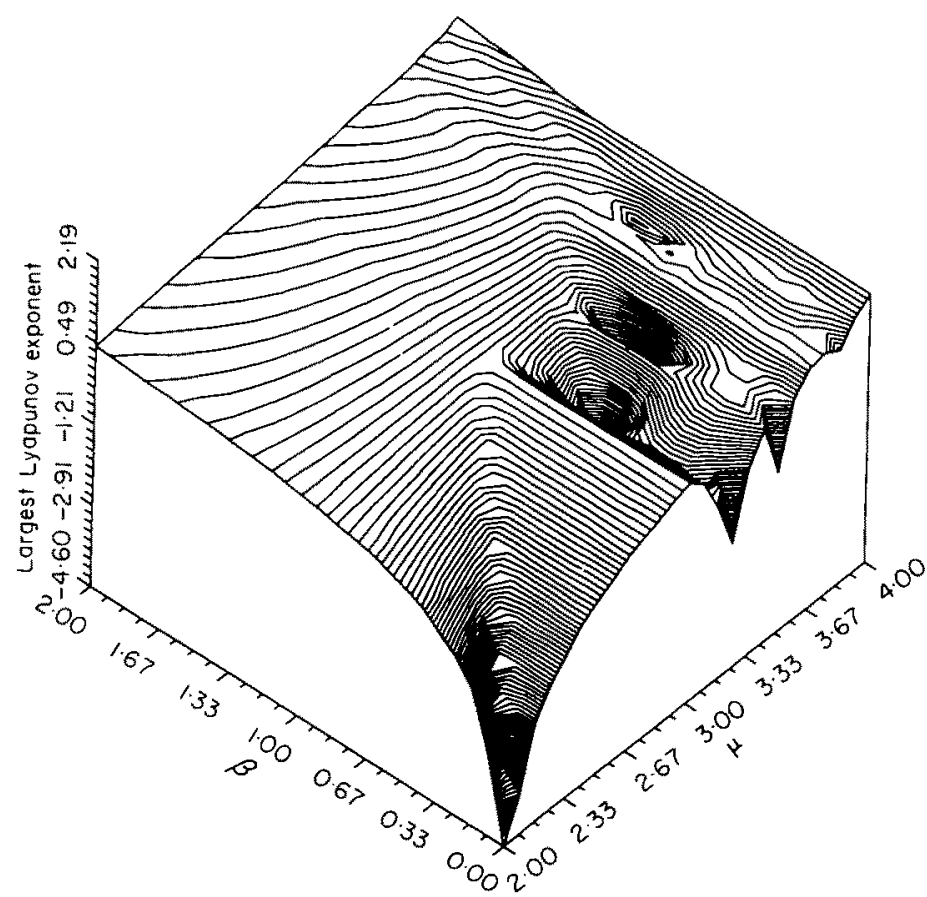

Fig. 1. Largest Lyapunov exponents $\left(\lambda_{m}\right)$ for model $(2)$ in relation with parameters $\mu$ and $\beta$. As can be seen, there are different stationary, periodic and chaotic attractors depending on the parameter values. Calculations were made using 2000 time steps after 1000 were discarded. The initial conditions were the same for all calculations. 


\section{Spatially Extended Model}

Space is now introduced using a discrete $N \times N$ lattice of points. The new set of equations (a CML) will be:

$$
\begin{aligned}
& x_{n+1}(\mathbf{k})=\mu x_{n}(\mathbf{k})\left(1-x_{n}(\mathbf{k})-\beta y_{n}(\mathbf{k})\right)+D_{1} \partial^{2} x_{n}(\mathbf{r}) \\
& y_{n+1}(\mathbf{k})=\mu y_{n}(\mathbf{k})\left(1-y_{n}(\mathbf{k})-\beta x_{n}(\mathbf{k})\right)+D_{2} \partial^{2} y_{n}(\mathbf{r})
\end{aligned}
$$

with $\mathbf{k}=(i, j)$ and where the coupling is defined as the diffusive operator:

$$
\partial^{2} x_{n}(\mathbf{r})=x_{n}(i, j+1)+x_{n}(i, j-1)+x_{n}(i+1, j)+x_{n}(i-1, j)-4 x_{n}(i, j)
$$

$D_{1}$ and $D_{2}$ are the diffusion rates. An additional rule is used in order to ensure nonnegative populations i.e. $x_{n}(i, j)=0$ if $x_{n}(i, j)<0$. Zero-flux boundary conditions are used.
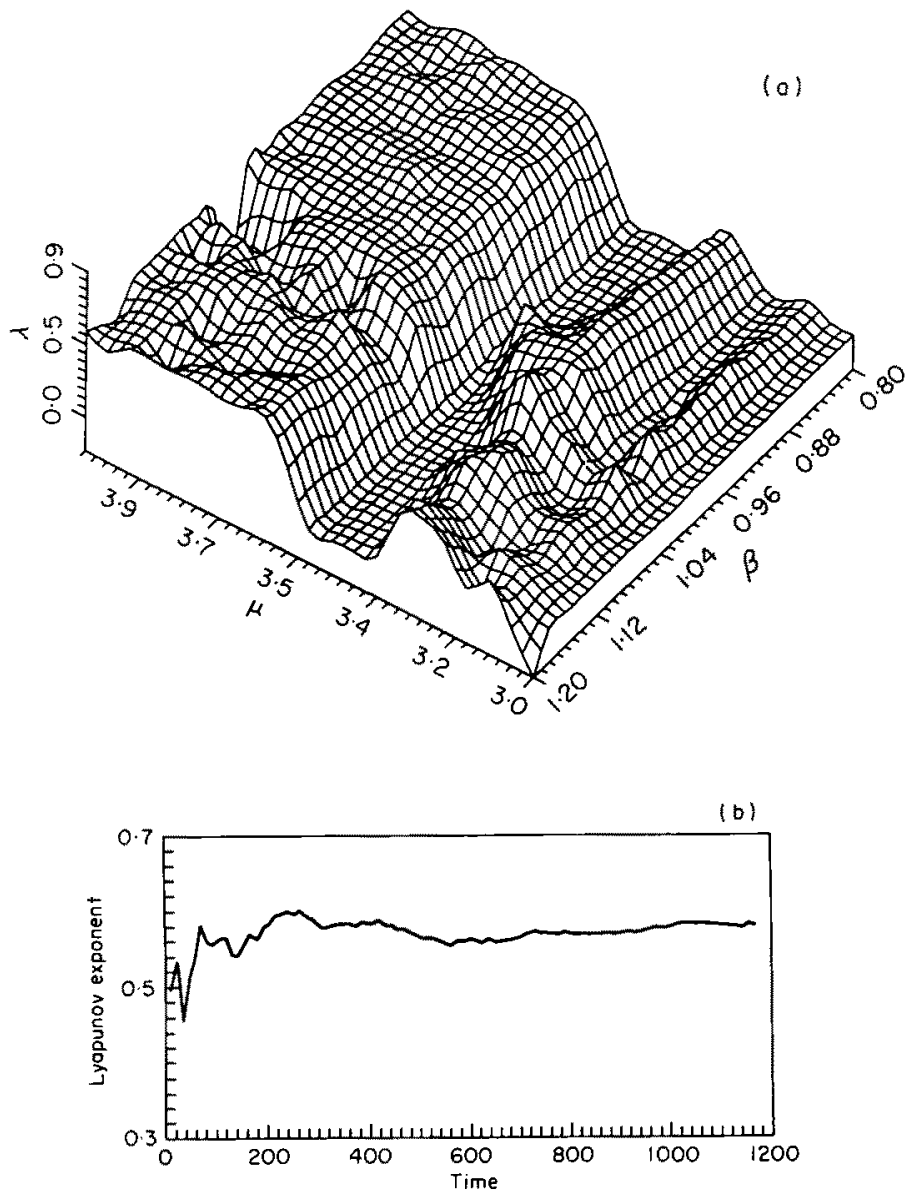

FIG. 2. (a) Similar to Fig. I but now for the CML counterpart. As can be observed for comparison with Fig. 1, the spatial degrees of freedom affect the dynamical properties of the system. Calculations were made using 1000 time steps after 500 were discarded. Lattice size is $30 \times 30$. (b) This shows the fast convergence of the Lyapunov exponents. 
Figure 2 shows the largest Lyapunov exponent for different parameter values for the CML counterpart. Positive exponents (indicating chaotic dynamics) are more extended than for model (2). In other words, parameter combinations that gave periodic attractors in model (2), now give chaotic attractors. It is an interesting example of diffusion-induced chaos (Kuramoto, 1984). When space is introduced, chaos become more frequent and robust and this has important consequences in ecology (Solé \& Valls, 1992).

In all the following calculations we introduce a high competition, i.e. $\beta=1 \cdot 2$. For $\beta<1$, low interspecific competition is present at all lattice points. In such situation, coexistence will be naturally present. For $\beta>1$, the high competition will lead, at least locally, to extinction of one of the competitors. The question is how this situation will be present in the spatially extended counterpart. As is shown in Fig. 3, there is
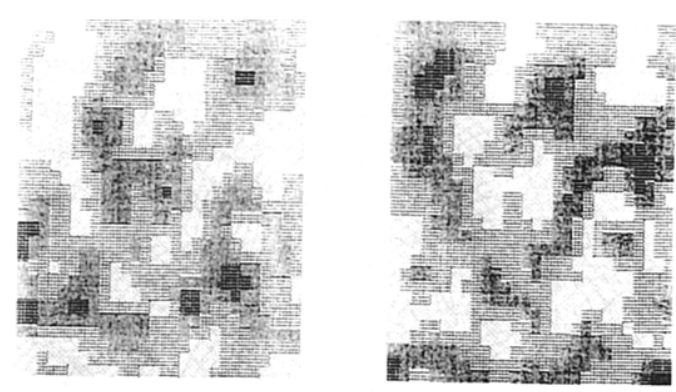

(a)
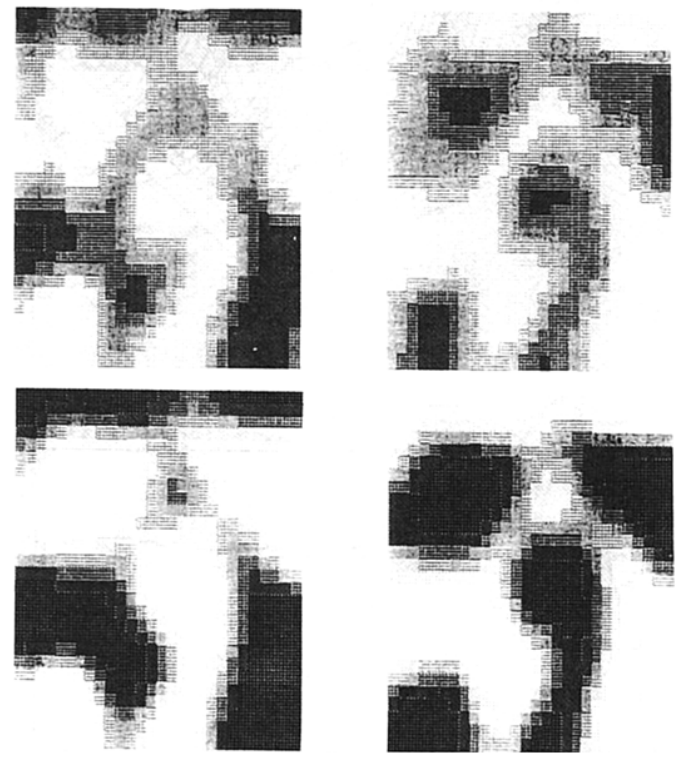

FIG. 3. Turing-like structures involving the local exclusion of the two competing species. One species is shown at the right-hand and the other at the left-hand. Three temporal states of the evolution are shown. From upper to bottom: initial configuration, an intermediate one and the final, a time invariant spatial pattern. In all cases $D=0.05, \beta=1 \cdot 2, N^{2}=30 \times 30$ and $\mu$ is respectively, (a) 2.5 corresponding to a temporal steady state and (b) 3.6 corresponding to chaotic dynamics. 
(b)
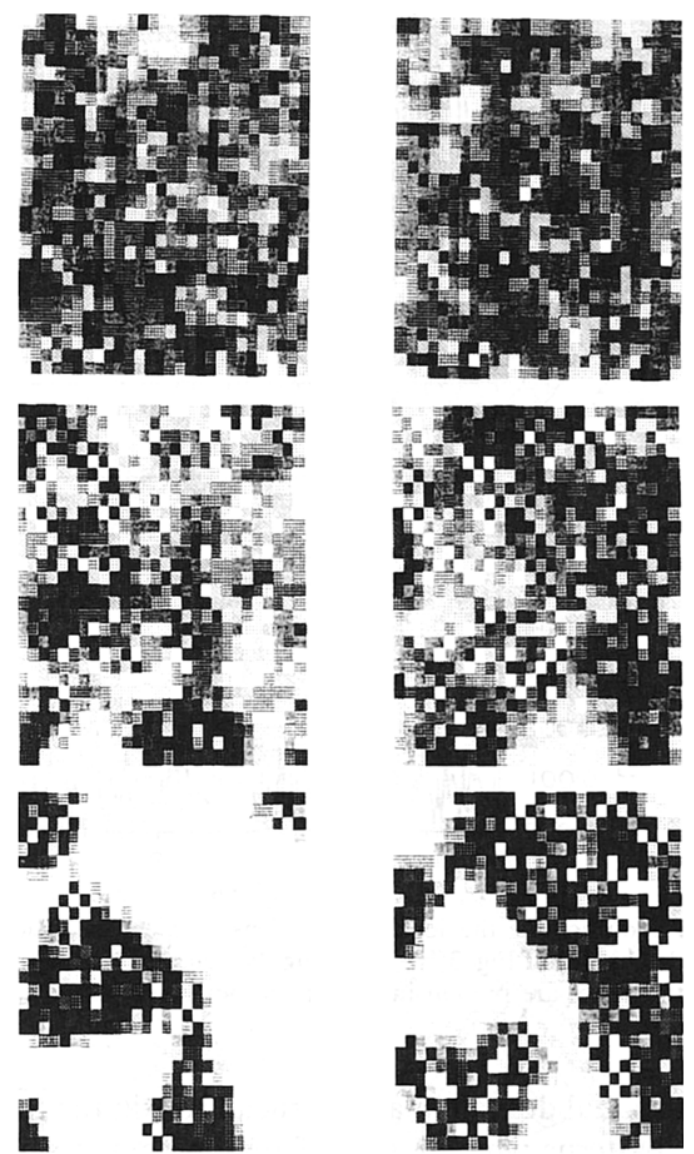

Fio. 3-Continued.

coexistence between the two species which is related with spatial structures. It is a new, unexpected result because, as we pointed out above, coexistence in model (2) is highly unstable for $\beta>1$. The structures formed are yet well defined after $n \approx 50$ generations and they are time invariant. This result is equivalent to those present in Turing structures (Turing, 1952) but there are two differences. First, while in Turing structures there is an explicit asymmetry in the equations' structure, in our case there is no asymmetry (there is neither "activator" nor "inhibitor"). On the other hand, these structures are formed even with a parameter combination that gives chaotic dynamics. This coexistence of order and chaos has been shown in previous papers (Sole \& Valls, 1991). The local dynamics of one of the lattice points of Fig. 3(b) can be seen in Fig. 4. There is a Lyapunov exponent of about 0.4 associated with this particular point. These Chaotic Turing Structures are a fairly common emergent property of our model for a wide range of parameter combinations. We have found an unexpected richness of spatiotemporal behaviour similar to that shown in a recent paper by Hassell et al. (1991) on host-parasitoid dynamics. 

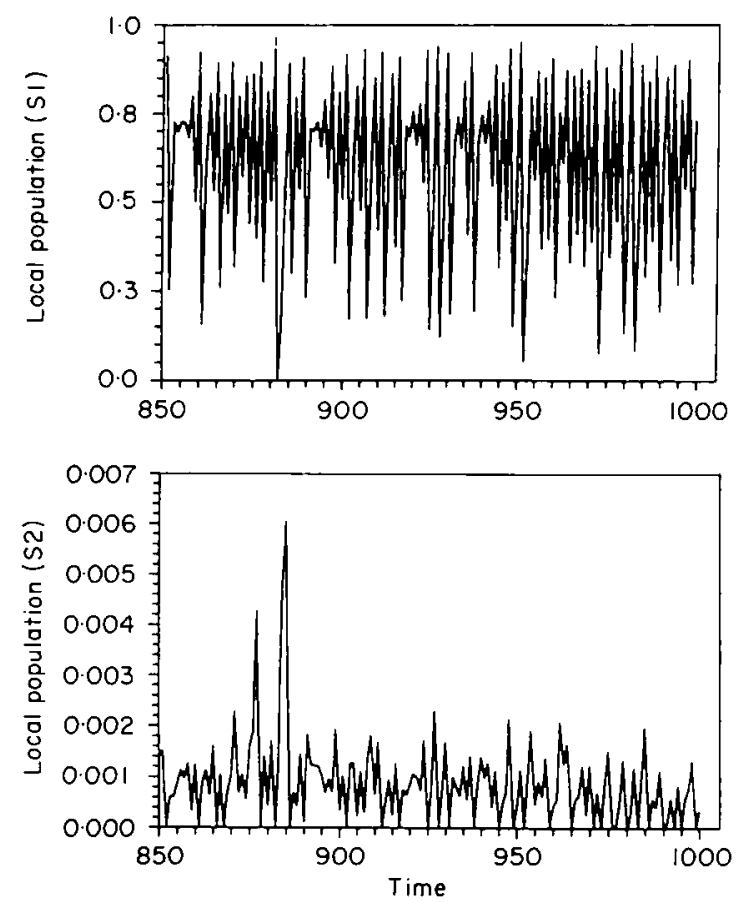

Fig. 4. Temporal evolution of the local populations at a given lattice point. The dynamics were computed for the node $(13,13)$ of Fig. $3($ b). These highly chaotic fluctuations are related with a Lyapunov exponent of 0.4 . Both species are present in this patch, but one of these is nearly extinct.

The size of the spatial domain plays a crucial role in the formation and persistence of the structural patterns which result. If the domain is small enough, only one of the competitors can persist. Increasing the size of space, a bifurcation point appears and we obtain two different patches in a similar way to that of Murray (1981). The bigger the spatial domain, the more frequent the number of patches. In this sense, the degree of complexity is given by the ratio of the diffusion rate to the size of the space. Increasing the lattice for a given diffusion rate has the same effect than decreasing the diffusion rate for a fixed lattice size (see Fig. 5).

Although the local dynamics are so unstable (with positive Lyapunov exponents), global dynamics are similar to those of a steady state with added noise. As seen in Fig. 6, global populations are nearly constant. We believe that this is a good argument against the point of view of authors such as Berryman \& Millstein (1989) who argue that chaotic dynamics can facilitate the probability that a population becomes extinct.

\section{Summary and Discussion}

Let us emphasize some of our conclusions by the following remarks:

(1) Our discrete time model for a two-species competition shows a wide range of dynamical behaviour, including chaos. 

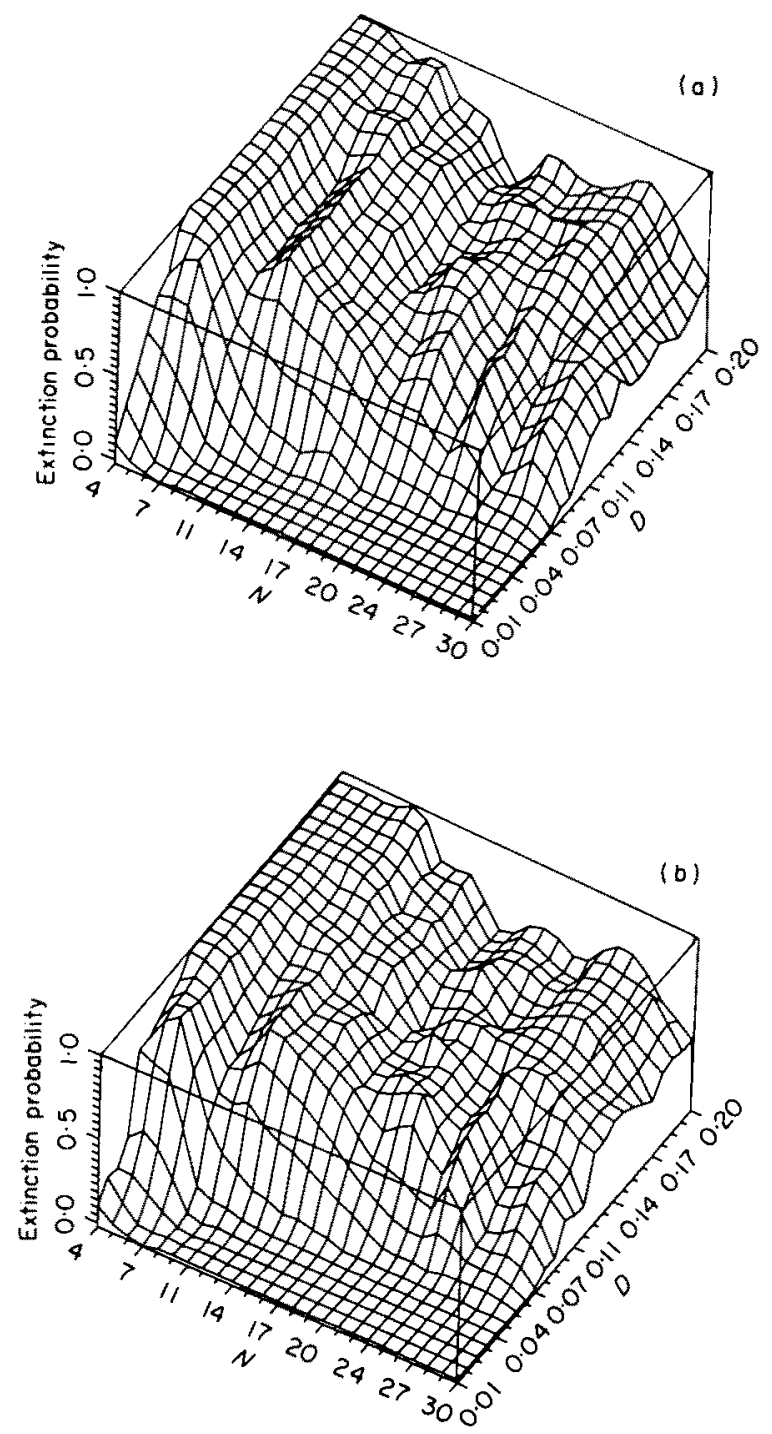

FIG. 5. Extinction probabilities for the competition CML, in relation to the lattice size and to the diffusion rate. The extinction of one of both species is given by the proportion of 20 replicates failing to persist over 2000 generations. As can be observed, the larger the space domain, the less the probability of extinction. (a) $\mu=3 \cdot 3$ and (b) $\mu=3 \cdot 6$.

(2) The spatially extended counterpart, based on the CML formalism, allows the global coexistence of the two species included for high enough values of interspecific competition.

(3) This coexistence implies the formation of structures over space with local segregation. 


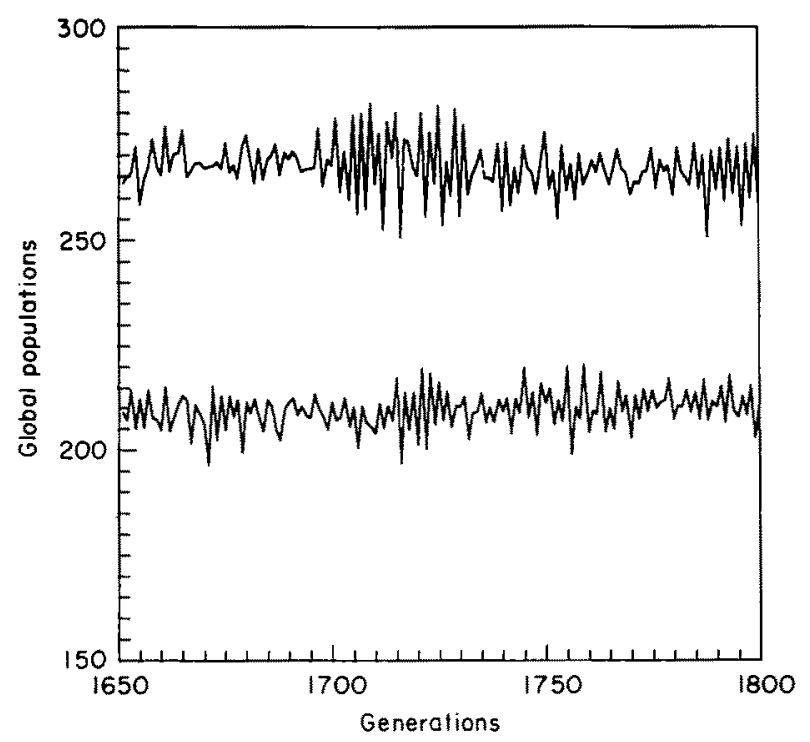

FIG. 6. Temporal dynamics of the global population of species 1 (below) and species 2 (above). For each time step we calculated the sum of both populations over all lattice points. Global populations are nearly constant in spite of the highly unstable local behaviour shown in Fig. $4 . \mu=3.6, N^{2}=30 \times 30, D=$ 0.05 and $\beta=1 \cdot 2$.

(4) The structural patterns can coexist with temporal chaotic dynamics.

(5) Although the local dynamics may be unstable, global populations are nearly constant.

Which is the mechanism of generation of these patches and which biological implications have they? Our spatially extended model holds the stability properties locally, i.e. on one lattice point only one of both species can coexist. The initial density is generated with some degree of stochasticity. In this sense, small differences in the numerical proportion of the competitors grow exponentially. The system evolves locally toward the extinction of one species and the global structural patterns which result from non-linear interactions are very sensitive to initial fluctuations, as can be seen in Fig. 7. This phenomenon can be common in nature, where the spatial pattern can be seen as the amplification of some initial distributions, the consequence of which is to hinder future changes and decrease the turnover (Margalef, 1980). The size of the patches depends on the diffusion and growth rates. If one species is more abundant at a point, it will also diffuse faster towards its neighbourhood and so it will increase there. The biological consequence of this spatial heterogeneity is to vary the importance of interspecific competition in front of an intraspecific one when we move along different spatial scales. Thus, if there is local competitive exclusion, globally there is coexistence because the outcome of the interaction is not the same in different points (patches). A practical consequence for the management and protection of natural resources is that an ecosystem cannot be shielded without protecting a large enough spatial domain. 

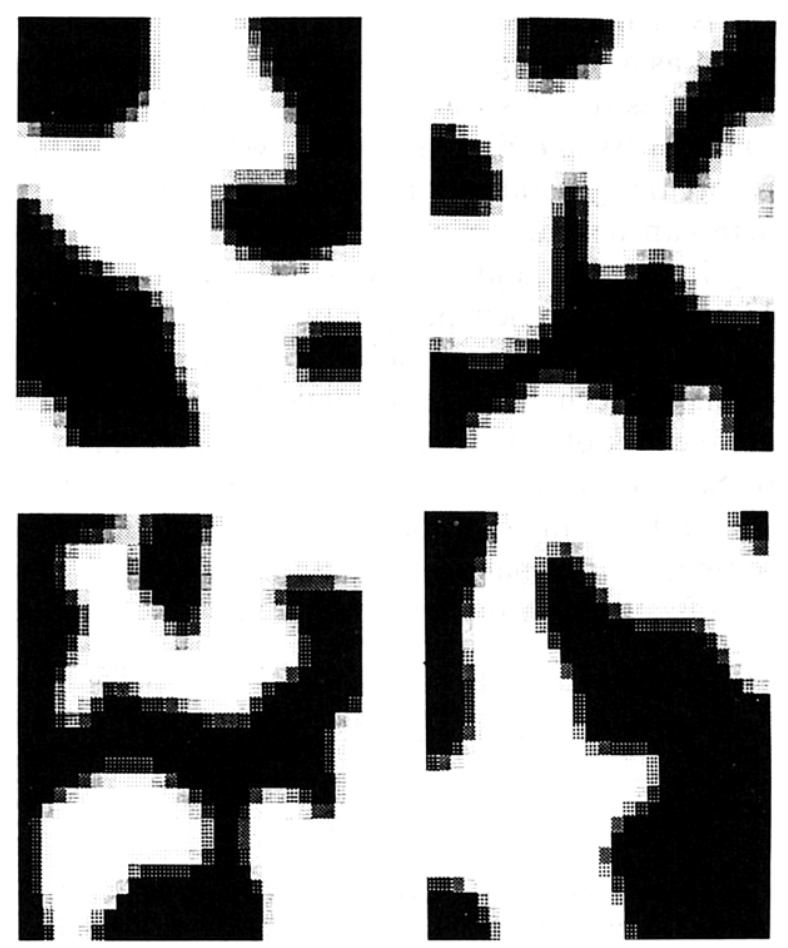

FIG. 7. Spatial patterns are very sensitive to initial conditions. All four final configurations correspond to the same parameter values $(\mu=2 \cdot 5, D=0.05$ and $\beta=1 \cdot 2)$ and the same species. These differences are, in fact, the amplifications of little initial fluctuations due to the non-linearity of interactions. The initial density over all lattice points is given by $Z_{0}(i, j)=0 \cdot 1+\xi$ for $Z=X, Y$. Here $\xi \in(0,0 \cdot 1)$ is randomly generated.

The global persistence of both competing species in spite of local exclusion is one of the most important results of our study. Interspecific competition being higher than intraspecific, this global stability (coexisting with local chaos) indicates that some ideas concerning niche theory should be reviewed. Hutchinson (1965) stated that coexistence is only possible if there is compartmentation of the fundamental niche, i.e. if there is some kind of ecological differences between the opponents. These restrictions can be relaxed only if some external variability or stochastic factors are considered. In other words, persistence needs a reduction of the interspecific competition pressure. A discussion about this point is made by Chesson (1991) and Shorrocks (1991) who questioned the need for niches. In the CML counterpart of our model we have shown spatial coexistence although the previous condition does not hold. Furthermore, in our simulation we have selected the most symmetrical case, i.e. two identical species. This result is in agreement with that of Shorrocks et al. (1984) showing that "Drosophila species will rarely exclude one another in nature even if they show no traditional resource partitioning and compete most strongly". 
Our conclusion is that coexistence would be easier when space is considered. Space can be viewed as a limiting resource. Its compartmentation under non-linear interactions corroborates the classical theory but, simultaneously, gives us a framework in which the richness and diversity of our world is possible.

We hope to report the generalization from 2 to $n$ species very soon. We believe that such a system can help us to understand the self-organization properties of ecological systems. In this way, old questions such as the existence of an upper limit to diversity could be seen from new perspectives. This theoretical framework invites the study of real temporal series, in spite of the fact that there are formidable problems in applying the techniques of dynamical system theory in ecology. Among these problems are the lack of long-term data and the high amount of noise (Godfray \& Blythe, 1990). So, we need the development of new techniques conceived for biology. Finally, it is important to consider the simulation of practical examples based on the Monte Carlo formalism, because in these kind of systems the experimentation is very difficult given the large temporal and spatial scale in which they take place.

This work has been supported by the grants of CIRIT EE91/1.

\section{REFERENCES}

Begon, M. \& Mortimer, M. (1986). Population Ecology. Oxford: Blackwell Scientific Publications. Berryman, A. A. \& Millstein, J. A. (1989). Trends Ecol. Evol. 4, 26-28.

Chesson, P. (1991). Trends Ecol. Evol. 6(1), 26-28.

Godfray, H. C. J. \& Blythe, S. P. (1990). Phil. Trans. R. Soc, Lond. B 330, $221-233$.

Hassell, M. P. \& Comins, H. N. (1976). Theor. Pop. Biol. 9(2), 202-220.

Hassell, M. P., Comins, H. N. \& May, R. M. (1991). Nature, Lond. 353, 255-258.

Hutchinson, E. (1965). The Ecological Theatre and the Evolutionary Play. New Haven, CT: Yale University Press.

Kaneko, K. (1990). Phys. Lett. 149A, 105-112.

Kuramoto, Y. (1984). Chemical Oscillations, Waves and Turbulence. Berlin: Springer.

LotKA, A. J. (1925). Elements of Physical Biology. Baltimore, MD: Williams and Wilkins.

Margalef, R. (1980). La Biosfera. Entre la Termodinámica y el Juego. Barcelona: Editorial Omega.

Margalef, R. (1986). Int. Symp. Long Term Changes Mar. Fish Pop. Vigo, 321-343.

MURRAY, J. D. (1981). J. theor. Biol. 88, 161-199.

Murray, J. D. (1989). Mathematical Biology. Berlin: Springer.

Nicolis. G. \& Prigogine, I. (1977). Self Organization in Nonequilibrium Systems. New York: Wiley.

Sabelis, M. W., Diekmann, O. \& Jansen, V. A. A. (1991). Biol. J. Limn. Soc. 42, 267-283.

Segel, L. A. \& Jackson, J. L. (1972). J. theor. Biol. 37, 545-559.

Shorrocks, B., Rosewell, J. \& Edwards, K. (1984). Nature, Lond. 310, 310-312.

SHORROCKS, B. (1991). Trends Ecol. Evol. 6(8), 262-263.

Sole, R. V. \& VALLS, J. (1991). Phys. Lett. 153A, 330-336.

SOLE, R. V. \& VALLS, J. (1992). J. theor. Biol. 155, 87-102.

TAYLOR, A. D. (1990). Ecology 71(2), 429-433.

Turing, A. (1952). Phil. Trans. R. Soc, Land. 237, 37-72.

VOLTERRA, V. (1926). Variations and fuctuations of the numbers of individuals in animal species living together. (Translation in Chapman, R. N., 1931, Animal Ecology, pp. 409-448, New York: McGraw Hill. 